\title{
Cálculo integral para o Ensino Médio
}

\author{
Alyrio A. Cordeiro Neto
}

\section{Resumo}

Nos exames do Programme for International Student Assessment (Pisa), há questões que avaliam a capacidade de o estudante estimar áreas de uma região do plano, onde a fronteira não é dada apenas por segmentos de reta, mediante as informações contidas num mapa. Motivados por esses problemas, apresentamos uma sequência didática para introduzir, no Ensino Médio, noções rudimentares do Cálculo Integral, com auxílio do software de geometria dinâmica, o GeoGebra, para que possamos obter as estimativas. Mostramos também que, por intermédio dos conhecimentos sobre os processos de integração, os estudantes da última etapa da Educação Básica podem desenvolver algumas competências e habilidades específicas de Matemática e suas tecnologias descritas na Base Nacional Comum Curricular (BNCC).

Palavras-chave: Cálculo Integral; Ensino Médio; GeoGebra; Área; Estimativa.

\section{Abstract}

In the examinations of the Program for International Student Assessment (Pisa), there are questions that assess the student's ability to estimate areas of a region of the plane, where the boundary is not given only by straight line segments, using the information contained in a map. Motivated by these problems, we present a didactic sequence to introduce, in High School, rudimentary notions of the Integral Calculus, with the aid of the software of dynamic geometry GeoGebra, so that we can obtain the estimates. We also show that through the knowledge about integration processes, students of the last stage of Basic Education can develop some specific skills and abilities of Mathematics and its technologies described in the National Curricular Common Base (BNCC).

Keywords: Integral Calculus; High school; GeoGebra; Area; Estimation

\section{Introdução}

Em janeiro de 2019, vimos que ocorreu um desastre em Brumadinho, região metropolitana de Belo Horizonte, em Minas Gerais, devido ao rompimento de uma barragem de rejeitos de minério de ferro de uma mineradora multinacional. A lama de rejeitos atingiu a área administrativa da mineradora, bairros e comunidades próximas, pousadas, áreas de cultivo, pastagens, além de estradas e vias rurais (ver figura 1 ). 


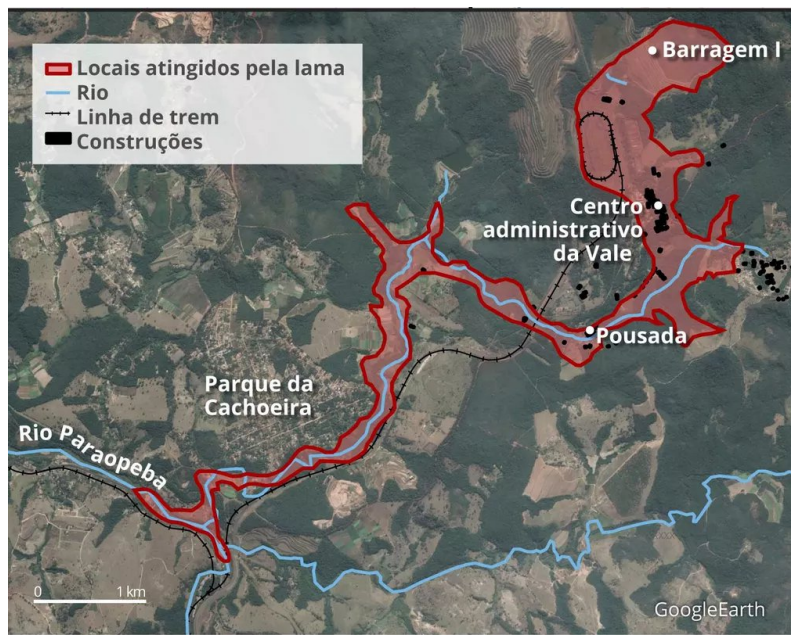

Figura 1: Caminho da lama após o rompimento da barragem em Brumadinho (MG) Fonte: [5]

Após essa tragédia, é do interesse dos ambientalistas e autoridades estimar a área devastada. Mas a região em evidência não é delimitada apenas por segmentos de retas. Então, qual o procedimento que eles podem recorrer para obter, aproximadamente, a área atingida?

Questões envolvendo estimativas da área de uma região do plano qualquer também fazem parte do exame do Pisa, ver [7], como exemplo, citamos: usando a escala do mapa (ver Figura 2), estime a área da Antártida e explique como você fez a estimativa.

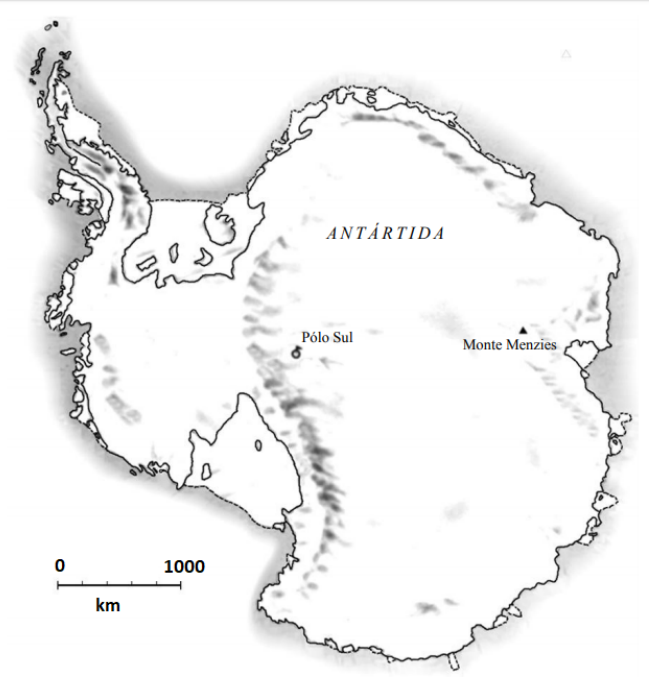

Figura 2: Mapa da Antártida Fonte: [7] 
Submetendo esses problemas aos alunos da Educação Básica, eles deverão procurar estratégias, conceitos, definições e procedimentos matemáticos para chegar a uma solução. E, de fato, essas expectativas estão de acordo com algumas competências e habilidades específicas de Matemática da Base Nacional Comum Curricular (BNCC) do Ensino Médio, ver [1].

Por outro lado, quando se conhece o Cálculo Integral, quase imediatamente pensa-se em obter as aproximações para as áreas das regiões solicitadas nesses problemas por meio da integral da diferença entre duas funções num intervalo fechado tais que as regiões estejam entre seus gráficos, como na Figura 3. Isso seria fácil por intermédio do aplicativo de geometria dinâmica, o GeoGebra, pois, ele oferece recursos para obtermos as funções necessárias e para determinar a integral definida num intervalo fechado qualquer.

Portanto, mediante o ensino dos processos de integração na etapa final da Educação Básica, podemos prover os alunos de ferramentas matemáticas para que possam propor soluções para esses problemas e estimular o desenvolvimento de competências e habilidades específicas de Matemática e suas tecnologias, conforme as descritas na BNCC.

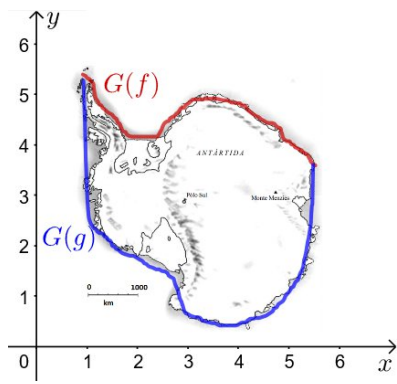

Figura 3: Mapa da Antártida entre os gráficos das funções $f$ e $g$ Fonte: Autoria própria

O objetivo deste artigo é mostrar a possibilidade da inclusão das noções básicas de integração nos programas da etapa final da Educação Básica de tal forma que tais conhecimentos ajudem a desenvolver as competências e habilidades específicas de Matemática da BNCC do Ensino Médio.

\section{Introdução ao Cálculo Integral}

Nesta seção abordaremos o seguinte problema: qual o valor da área da região $A$ (ver Figura 4), onde $f$ é uma função positiva? 


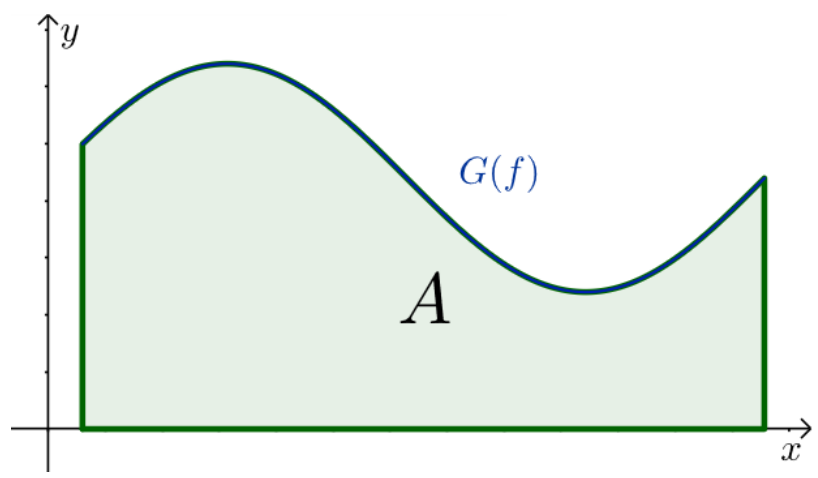

Figura 4: Região $A$

Fonte: Autoria própria

A solução desse problema será dada em quatro etapas. Nas três primeiras etapas, obteremos aproximações para a área da região $A$ e, na última, apresentaremos a solução. Essas etapas serão descritas mediante a solução da questão seguinte.

Problema 1. Seja $f:[0,40] \rightarrow \mathbb{R}$ uma função, definida por $f(x)=-\frac{x^{2}}{10}+250$. Qual a área da região $A$ (ver Figura 5 ), sob o gráfico de $f$ ?

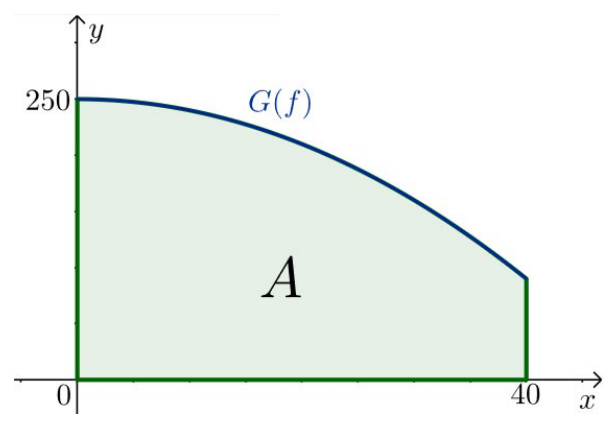

Figura 5: Região $A$ sob gráfico de $f:[0,40] \rightarrow \mathbb{R}$ definida por $f(x)=-\frac{x^{2}}{10}+250$ Fonte: Autoria própria

Notemos que a fronteira da região $A$ não é dada apenas por segmentos de retas. Ou seja, não será possível determinar a área de $A$ mediante os métodos presentes nos programas da Educação Básica. Por isso, descreveremos uma estratégia para obter aproximações para $A$ e, em seguida, resolveremos esse problema.

Inicialmente, divida o intervalo $[0,40]$ em quatro subintervalos $I_{i}$ de mesmo comprimento, isto é, $I_{i}=[10(i-1), 10 i], i=1,2,3,4$, e considere os retângulos sobre cada intervalo $I_{i}$ de altura $f(10 i)$, como na Figura 6. 


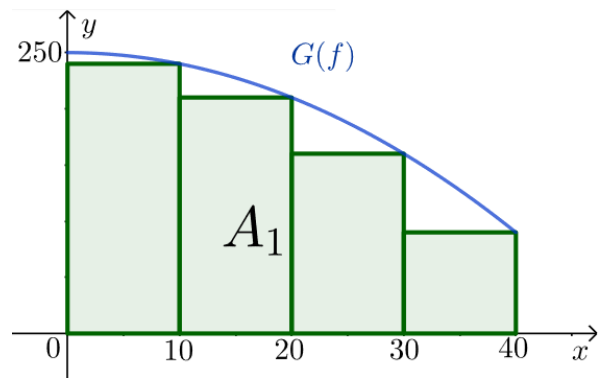

Figura 6: Retângulos sobre cada intervalo $[10(i-1), 10 i]$ de altura $f(10 i)$ Fonte: Autoria própria

Note que, de modo impreciso, a área da região $A_{1}$ é próxima da área da região $A$, isto é,

$$
\operatorname{Área}(A) \cong \operatorname{Área}\left(A_{1}\right)=\sum_{i=1}^{4}\left[\frac{40}{4} f\left(\frac{40}{4} i\right)\right]=10 \sum_{i=1}^{4} f(10 i)=7000 \text {. }
$$

Para uma melhor estimativa, divida o intervalo $[0,40]$ em, por exemplo, dez subintervalos $I_{i}$ de mesmo comprimento, isto é, $I_{i}=[4(i-1), 4 i], i=1,2, \ldots, 9,10$, e considere os retângulos sobre cada intervalo $I_{i}$ de altura $f(4 i)$, como na Figura 7 .

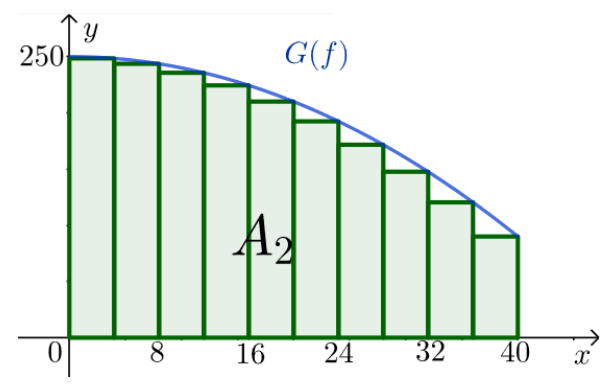

Figura 7: Retângulos sobre cada intervalo $[4(i-1), 4 i]$ de altura $f(4 i)$ Fonte: Autoria própria

Observe que, de fato, a área da região $A_{2}$ é uma aproximação melhor para a área da região $A$. Dessa forma,

$$
\operatorname{Área}(A) \cong \operatorname{Area}\left(A_{2}\right)=\sum_{i=1}^{10}\left[\frac{40}{10} f\left(\frac{40}{10} i\right)\right]=4 \sum_{i=1}^{10} f(4 i)=7536 .
$$

Para obter uma estimativa ainda melhor do que os resultados obtidos nas Equações (1) e (2) para área de $A$, divida o intervalo $[0,40]$ em um número maior de subintervalos $I_{i}$ de mesmo comprimento. Por exemplo, divida em vinte subintervalos, isto é, $I_{i}=[2(i-1), 2 i], i=1,2, \ldots, 19,20$, e considere os retângulos sobre cada intervalo $I_{i}$ de altura $f(2 i)$, como na Figura 8 . 


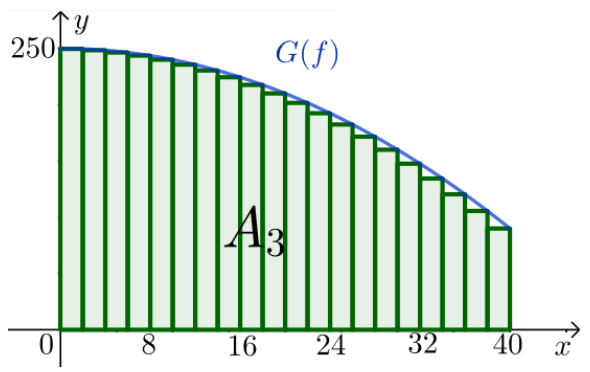

Figura 8: Retângulos sobre cada intervalo $[2(i-1), 2 i]$ de altura $f(2 i)$

Fonte: Autoria própria

Intuitivamente, podemos afirmar que a área da região $A_{3}$ é uma aproximação melhor para a área de $A$ do que as áreas de $A_{1}$ e $A_{2}$. Então,

$$
\text { Área }(A) \cong \operatorname{Area}\left(A_{3}\right)=\sum_{i=1}^{20}\left[\frac{40}{20} f\left(\frac{40}{20} i\right)\right]=2 \sum_{i=1}^{20} f(2 i)=7704 \text {. }
$$

Note que obtivemos as estimativas para a área da região $A$ mediante o cálculo das áreas das regiões $A_{1}, A_{2}$ e $A_{3}$. Então, deduzimos, intuitivamente, que as aproximações para a área de $A$ são dadas por

$$
\operatorname{Area}\left(A_{n}\right)=\sum_{i=1}^{n}\left[\frac{40}{n} f\left(\frac{40}{n} i\right)\right]
$$

onde $n$ é o número de subintervalos, de mesmo comprimento, do intervalo $[0,40]$.

Portanto,

$$
\begin{aligned}
\text { Área }(A) \cong \text { Área }\left(A_{n}\right) & =\sum_{i=1}^{n}\left[\frac{40}{n} f\left(\frac{40}{n} i\right)\right]=\frac{40}{n} \sum_{i=1}^{n} f\left(\frac{40}{n} i\right) \\
& =\frac{40}{n} \sum_{i=1}^{n}\left[-\frac{\left(\frac{40}{n} i\right)^{2}}{10}+250\right]=\frac{40}{n}\left[-\sum_{i=1}^{n} \frac{\left(\frac{40}{n} i\right)^{2}}{10}+250 n\right] \\
& =\frac{40}{n}\left[-\frac{160}{n^{2}} \sum_{i=1}^{n} i^{2}+250 n\right]=10000-\frac{6400}{n^{3}} \sum_{i=1}^{n} i^{2} .
\end{aligned}
$$

Observe que para determinarmos a área de $A_{n}$, precisamos calcular o valor de $\sum_{i=1}^{n} i^{2}$. Então, assumiremos o fato que

$$
\sum_{i=1}^{n} i^{2}=\frac{n(n+1)(2 n+1)}{6}
$$

cuja demonstração pode ser encontrada em [2, p16] 
Desse forma,

$$
\begin{aligned}
\operatorname{Area}\left(A_{n}\right) & =10000-\frac{6400}{n^{3}} \frac{n(n+1)(2 n+1)}{6} \\
& =12500-\left(\frac{6400}{3}+\frac{3200}{n}+\frac{3200}{3 n^{2}}\right) \\
& =\frac{23600}{3}-\frac{3200}{n}-\frac{3200}{3 n^{2}}
\end{aligned}
$$

Por último, nota-se, intuitivamente, que a área de $A_{n}$ aproxima-se da área de $A$ tanto quanto se queira, bastando tornar $n$ cada vez maior. Ou seja,

$$
\operatorname{Area}(A)=\lim _{n \rightarrow+\infty} \operatorname{Area}\left(A_{n}\right)=\lim _{n \rightarrow+\infty}\left(\frac{23600}{3}-\frac{3200}{n}-\frac{3200}{3 n^{2}}\right)=\frac{23600}{3} .
$$

Vale observar que a estratégia de aumentar indefinidamente o número de retângulos na região sob o gráfico de uma função para determinar sua área é muito semelhante ao método utilizado por Antífton (c. 430 a. C.), um contemporâneo de Sócrates, para o problema da quadratura do círculo, ver [3]. Ele teria antecipado a ideia de que, por sucessivas duplicações do número de lados de um polígono regular inscrito num círculo (ver a Figura 9), a diferença final entre o círculo e o polígono exaurir-se-ia.

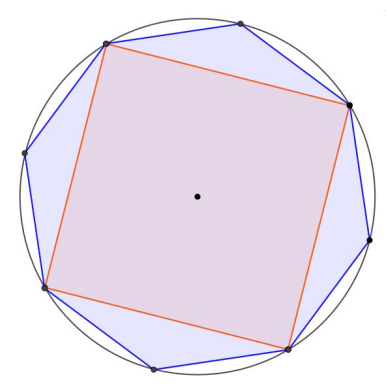

Figura 9: Polígonos regulares inscritos no circulo

Fonte: Autoria própria

Essa resolução apresentada por Antífton para o problema da quadratura do círculo contém o princípio do método da exaustão grego, cuja base é a seguinte proposição: se de uma grandeza qualquer subtrai-se uma parte não menor que sua metade, do restante subtrai-se também uma parte não menor que sua metade, e, assim por diante, chega-se por fim a uma grandeza menor que qualquer outra predeterminada da mesma espécie.

Os gregos, por volta de 300 a. C., na época que Euclides escrevia Os Elementos, estavam próximos da descoberta do Cálculo. Para avaliar até que ponto chegaram, basta verificar que Arquimedes (287-212 a. C.), em sua obra O Método, realizou o cálculo da área de um segmento parabólico (ver Figura 10), antecipando-se, assim, em mais de dezessete séculos aos resultados do Cálculo Integral. 


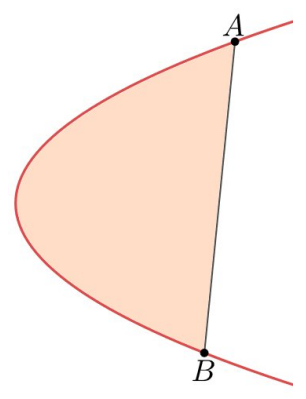

Figura 10: Segmento parabólico

Fonte: Autoria própria

O surgimento do Cálculo, no século dezessete, está em plena conexão com a busca de meios de simplificar os métodos gregos. Nesse século, a comunidade científica procurava respostas para algumas questões, como: determinar retas tangentes a curvas, resolver problemas de otimização e calcular áreas, volumes e centros de gravidade. Chegar a uma solução para esses problemas mediante o método da exaustão exigia muita engenhosidade. Então, alguns matemáticos enfrentaram o problema e deram suas contribuições. Newton e Liebniz, por exemplo, deram notórias contribuições, tanto que são considerados os criadores do Cálculo.

Vale destacar ainda a contribuição dada por Riemann para o Cálculo no século dezenove. Ele tornou claro o conceito de integrabilidade mediante definição do que denominamos atualmente como Integral de Riemann. Entretanto, a sua contribuição ao Cálculo é considerada pequena quando comparada com a dada em outras áreas, como na Geometria, cujo trabalho foi fundamental para a Teoria da Relatividade de Einstein, por exemplo.

No caso do problema do início desta seção, usamos os princípios do conceito de integral de Riemann para resolução. Então, definiremos formalmente esse conceito para que possamos aplicar em casos mais gerais.

\subsection{Integral de Riemann}

No caso particular do Problema 1, dividimos o intervalo [0,40] em alguns subintervalos. A partir de agora, usaremos o conceito de partição para isso.

Definição 1 (Partição). Uma partição de um intervalo $[a, b]$ é um conjunto $\mathcal{P}=\left\{x_{0}, x_{1}, x_{2}, \ldots\right.$, $\left.x_{n-1}, x_{n}\right\}$, com $x_{i} \in[a, b], i=1,2, \ldots, n-1, n$ e $a=x_{0}<x_{1}<x_{2}<\ldots<x_{n-1}<x_{n}=b$.

Exemplo 1. No Problema 1, usamos as partições $\mathcal{P}_{1}=\{0,10,20,30,40\}, \mathcal{P}_{2}=\{0,4,18, \ldots, 36,40\}$ e $\mathcal{P}_{3}=\{0,2,4, \ldots, 38,40\}$ do intervalo $[0,40]$.

As partições mostradas no Exemplo 1 são equiespaçadas, isto é, a diferença $\Delta_{i} x=x_{i}-x_{i-1}$, $i=1,2, \ldots, n-1, n$, é constante. Nesse caso, diremos que a norma da partição é igual a $\Delta_{i} x$. Mas, como uma partição não precisa ser necessariamente equiespaçada, definiremos sua norma de um modo geral. 
Definição 2 (Norma da partição). Chamamos norma da partição $\mathcal{P}$ o número $|\mathcal{P}|$, máximo do conjunto $\left\{\Delta_{1} x, \Delta_{2} x, \ldots, \Delta_{n-1} x, \Delta_{n} x\right\}$, onde $\Delta_{i} x=x_{i}-x_{i-1}, i=1,2, \ldots, n-1, n$.

Exemplo 2. Sejam $\mathcal{P}_{1}, \mathcal{P}_{2}$ e $\mathcal{P}_{3}$ as partições definidas no Exemplo 1. Logo, note que $\left|\mathcal{P}_{1}\right|=10$, $\left|\mathcal{P}_{2}\right|=5$ e $\left|\mathcal{P}_{3}\right|=2$.

Exemplo 3. Seja $\mathcal{P}=\left\{0, \frac{1}{3}, \frac{1}{2}, \frac{9}{10}, 1\right\}$ uma partição do intervalo $[0,1]$. Então, $|\mathcal{P}|=\frac{2}{5}$.

Observação 1 . Note que $\Delta_{i} x=x_{i}-x_{i-1}, i=1,2, \ldots, n-1, n$, representa, geometricamente, a distância entre os pontos $x_{i-1}$ e $x_{i}$. Ou seja, a norma $|\mathcal{P}|$ será a maior distância entre dois pontos consecutivos da partição $\mathcal{P}=\left\{x_{0}, x_{1}, x_{2}, \ldots, x_{n-1}, x_{n}\right\}$ de um intervalo $[a, b]$.

No caso do Exemplo 3, observa-se que a maior distância entre dois pontos consecutivos é, de fato, $\frac{2}{5}$ (ver Figura 11).

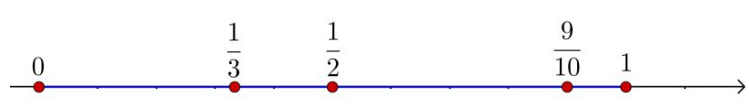

Figura 11: Representação geométrica da partição $\mathcal{P}=\left\{0, \frac{1}{3}, \frac{1}{2}, \frac{9}{10}, 1\right\}$ do intervalo $[0,1]$ Fonte: Autoria própria

Iniciamos a resolução do Problema 1 obtendo estimativas para a área da região $A$. Para isso, tomamos uma partição equiespaçada $\mathcal{P}=\left\{x_{0}, x_{1}, x_{2}, \ldots, x_{n-1}, x_{n}\right\}$ do intervalo [0,40], escolhemos a imagem de $\bar{x}_{i}=x_{i}, i=1,2, \ldots, n-1, n$, como a altura dos retângulos sobre esses subintervalos e, por último, determinamos a soma $\sum_{i=1}^{n} f\left(\bar{x}_{i}\right) \Delta_{i} x$ como uma aproximação para $A$. Esse somatório é o que denominamos de soma de Riemann e definiremos, de modo geral, a seguir.

Definição 3 (Soma de Riemann). Seja $\bar{x}_{i} \in\left[x_{i-1}, x_{i}\right], i=1,2, \ldots, n-1, n$, escolhido arbitrariamente nesse intervalo. Denominamos

$$
\sum_{i=1}^{n} f\left(\bar{x}_{i}\right) \Delta_{i} x=f\left(\bar{x}_{1}\right) \Delta_{1} x+f\left(\bar{x}_{2}\right) \Delta_{2} x+\ldots+f\left(\bar{x}_{n-1}\right) \Delta_{n-1} x+f\left(\bar{x}_{n}\right) \Delta_{n} x
$$

de soma de Riemann de $f$ em $[a, b]$ relativa à partição $\mathcal{P}$ e à escolha feita dos $\bar{x}_{i}$.

Geometricamente, o somatório na Equação (4) representa, ver Figura 12, a soma das áreas dos retângulos de bases $\Delta_{i} x$ e alturas $f\left(\bar{x}_{i}\right)$. 


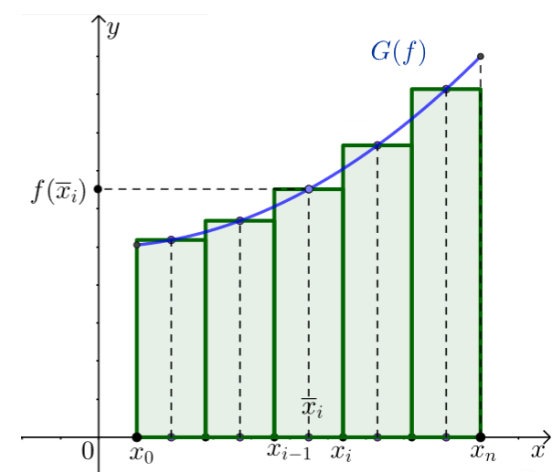

Figura 12: Representação geométrica da soma de Riemann Fonte: Autoria própria

Finalmente, para obter a solução do Problema 1, calculamos o limite, quando $n$ tende ao infinito, da soma de Riemann de $f$ em $[0,40]$ relativa a uma partição $\mathcal{P}$, pois, dessa forma, a soma tende à área de $A$, isto é,

$$
\operatorname{Area}(A)=\lim _{n \rightarrow+\infty} \sum_{i=1}^{n} f\left(\bar{x}_{i}\right) \Delta_{i} x .
$$

Nesse caso particular, note que se $n$ tende a $+\infty$, então (ver Figura 13), $|\mathcal{P}|$ tende a 0 . E , observe ainda que se $|\mathcal{P}|$ tende a 0 , então

$$
\operatorname{Area}(A)=\lim _{|\mathcal{P}| \rightarrow 0} \sum_{i=1}^{n} f\left(\bar{x}_{i}\right) \Delta_{i} x .
$$
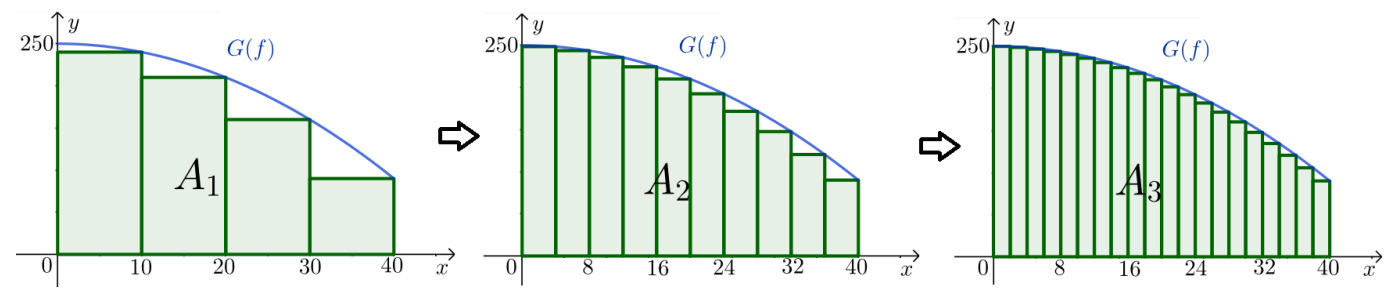

Figura 13: Representação geométrica da Integral no sentido de Riemann Fonte: Autoria própria

Nesse caso, se existir e for finito o limite da Equação (5), diremos que a função $f$ é integrável no sentido de Riemann e que a integral é igual a esse limite, isto é, igual à área de $A$.

Definição 4 (Função integrável no sentido de Riemann). Seja $f$ uma função contínua no intervalo fechado $[a, b]$. Diremos que $f$ é integrável no sentido de Riemann (ou apenas integrável) se existir e for finito o limite da soma de Riemann $\sum_{i=1}^{n} f\left(\bar{x}_{i}\right) \Delta_{i} x$ quando a norma da partição $\mathcal{P}$ tender a zero, isto é, se existir um número real $I$ satisfazendo a seguinte condição:

$$
\lim _{|\mathcal{P}| \rightarrow 0} \sum_{i=1}^{n} f\left(\bar{x}_{i}\right) \Delta_{i} x=I .
$$


Nesse caso, diremos que $I$ é a integral definida (ou apenas integral) de $f$ em $[a, b]$.

Observação 2. Denotaremos a integral definida $I$ usando a notação, devida a Liebniz:

$$
\int_{a}^{b} f(x) d x=\lim _{|\mathcal{P}| \rightarrow 0} \sum_{i=1}^{n} f\left(\bar{x}_{i}\right) \Delta_{i} x .
$$

Observação 3. Assumiremos que se a função $f$ é contínua no intervalo $[a, b]$, então $f$ é integrável em $[a, b]$.

Para verificar esse resultado, o leitor pode consultar [8, p. 131].

Vimos que o cálculo de áreas motiva conceito de Integral definida. Então, faz sentido definirmos a área da região compreendida entre o gráfico de uma função contínua num dado intervalo e o eixo das abcissas em relação ao cálculo da Integral.

Definição 5. Seja $A$ a região compreendida entre as retas $x=a, x=b$ e $y=0$, e o gráfico da função $f:[a, b] \rightarrow \mathbb{R}$ (ver Figura 14). Se $f$ é uma função integrável e não negativa, definimos a área da região $A$ sendo

$$
\operatorname{Area}(A)=\int_{a}^{b} f(x) d x .
$$

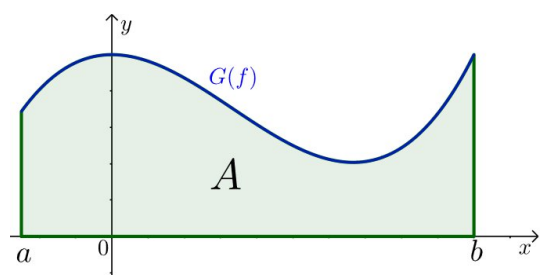

Figura 14: Área sob o gráfico da função $f:[a, b] \rightarrow \mathbb{R}$ tal que $f(x) \geq 0$ Fonte: Autoria própria

No Problema 1, conseguimos obter a área da região $A$ por intermédio do resultado que assumimos na Equação (3). Mas, como resolver esse problema sem assumir esse fato?

Embora tenhamos definido a Integral de modo geral, não elaboramos uma maneira de determiná-la. Por isso, apresentaremos um teorema a seguir que nos ajudará nessa questão.

Teorema 1 (Teorema Fundamental do Cálculo). Seja $f$ uma função contínua no intervalo $[a, b]$ e não negativa nesse intervalo. Se A é a função que associa a área sob o gráfico de $f$ no intervalo $[a, x]$, isto é,

$$
A(x)=\int_{a}^{x} f(t) d t,
$$

então $A$ é derivável em $(a, b)$ e $A^{\prime}(x)=f(x)$.

Observação 4. Ao longo desse artigo, assumiremos o seguinte teorema, devido a Weierstrass: se $f$ é uma função contínua no intervalo $[a, b]$, então existem $x_{m}, x_{M} \in[a, b]$ tais que $x_{m}$ é um mínimo global e $x_{M}$ é um máximo global.

O leitor pode consultar a demonstração desse resultado em [8, p82]. 
Demonstração. Sejam $x \in[a, b]$ e $h>0 \operatorname{com} x+h \in[a, b]$.

Se $f$ é contínua em $[a, b]$, por hipótese, então $f$ é contínua em $[x, x+h]$. Portanto, o teorema de Weierstrass garante que $f$ admite um ponto de máximo $x_{M}$ e um ponto de mínimo $x_{m}$ em $[x, x+h]$. Raciocinando geometricamente, em termos de área, ver Figura 15, segue que

$$
f\left(x_{m}\right) h \leq A(x+h)-A(x) \leq f\left(x_{M}\right) h .
$$

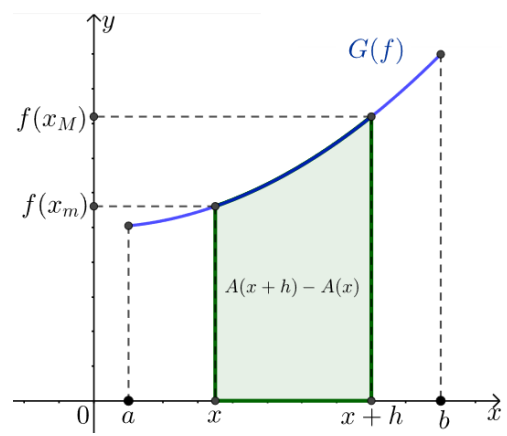

Figura 15: Área sob gráfico da função $f$ no intervalo $[x, x+h]$ Fonte: Autoria própria

Logo,

$$
f\left(x_{m}\right) \leq \frac{A(x+h)-A(x)}{h} \leq f\left(x_{M}\right) .
$$

Note que quando $h$ tende a zero, $f\left(x_{m}\right)$ e $f\left(x_{M}\right)$ se aproximam simultaneamente de $f(x)$ enquanto o quociente $\frac{A(x+h)-A(x)}{h}$ aproxima-se do $\operatorname{limite~} \lim _{h \rightarrow 0^{+}} \frac{A(x+h)-A(x)}{h}$, ou seja,

$$
f(x) \leq \lim _{h \rightarrow 0^{+}} \frac{A(x+h)-A(x)}{h} \leq f(x) .
$$

Então,

$$
f(x)=\lim _{h \rightarrow 0^{+}} \frac{A(x+h)-A(x)}{h} .
$$

Analogamente, quando $h<0$, considerando o intervalo $[x+h, x]$ (ver Figura 16), temos

$$
f\left(x_{m}\right)(-h) \leq A(x)-A(x+h) \leq f\left(x_{M}\right)(-h) .
$$

Logo,

$$
f\left(x_{m}\right) \leq \frac{A(x+h)-A(x)}{h} \leq f\left(x_{M}\right) .
$$

Como no caso anterior, se $h$ tende a zero, então $f\left(x_{m}\right)$ e $f\left(x_{M}\right)$ se aproximam simultaneamente de $f(x)$ enquanto o quociente $\frac{A(x+h)-A(x)}{h}$ aproxima-se do $\operatorname{limite}_{h \rightarrow 0^{-}} \frac{A(x+h)-A(x)}{h}$, isto é,

$$
f(x) \leq \lim _{h \rightarrow 0^{-}} \frac{A(x+h)-A(x)}{h} \leq f(x) .
$$




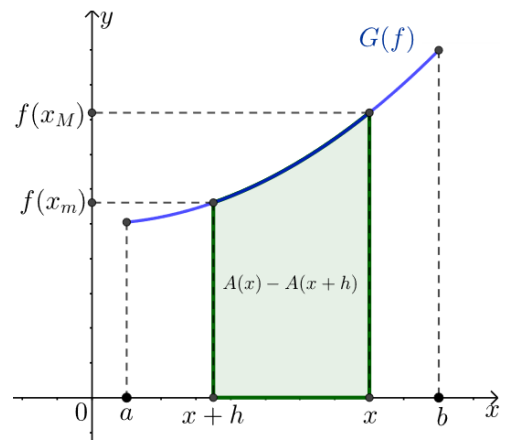

Figura 16: Área sob gráfico da função $f$ no intervalo $[x+h, x]$

Fonte: Autoria própria

Então,

$$
f(x)=\lim _{h \rightarrow 0^{-}} \frac{A(x+h)-A(x)}{h} .
$$

Portanto, $A$ é derivável em $(a, b)$ e $A^{\prime}(x)=f(x)$.

Definição 6 (Primitiva). Sejam $I \subset \mathbb{R}$ um intervalo e $f: I \rightarrow \mathbb{R}$ uma função integrável em todo intervalo $[a, b] \subset I$. Uma primitiva para $f$ em $I$ é uma função derivável $F: I \rightarrow \mathbb{R}$ tal que $F^{\prime}(x)=f(x)$ em $I$.

Exemplo 4. Seja $f: \mathbb{R} \rightarrow \mathbb{R}$ uma função, definida por $f(x)=x^{2}$. A função $F: \mathbb{R} \rightarrow \mathbb{R}$, definida por $F(x)=\frac{x^{3}}{3}+1$, é uma primitiva de $f$.

Exemplo 5. Seja $f: \mathbb{R} \rightarrow \mathbb{R}$ uma função, definida por $f(x)=x^{n}, n \in \mathbb{N}$. A função $F: \mathbb{R} \rightarrow \mathbb{R}$, definida por $F(x)=\frac{x^{n+1}}{n+1}$, é uma primitiva de $f$.

Observação 5. Por intermédio dos resultados do Cálculo diferencial, ver [6], mostra-se que se duas funções $f, g$ possuem a mesma derivada em um intervalo aberto $I$, então $f=g+c$, onde $c$ é uma constante.

Corolário 1. Sejam $f$ uma função contínua no intervalo $[a, b]$ e não negativa nesse intervalo, $F$ uma primitiva de $f$ e $A$ a função que associa a área sob o gráfico de $f$ no intervalo $[a, x]$, isto é,

$$
A(x)=\int_{a}^{x} f(t) d t .
$$

Então,

$$
\int_{a}^{b} f(t) d t=F(b)-F(a) .
$$

Demonstração. Raciocinando geometricamente, em termos de áreas, nota-se que $A(a)=\int_{a}^{a} f(t) d t=$ 0 e $A(b)=\int_{a}^{b} f(t) d t$ 
Por outro lado, se $A^{\prime}(x)=f(x)$, então $F(x)=A(x)+c$ visto que $F$ é uma primitiva de $f$. Logo, $F(a)=A(a)+c=c$ e $F(b)=A(b)+c$.

Portanto, $F(b)=A(b)+F(a)$.

Finalmente, por intermédio do Corolário 1, poderemos determinar a área da região $A$, do Problema 1, sem que seja necessário assumir o resultado da Equação (3).

Note que a função $F:[0,40] \rightarrow \mathbb{R}$, definida por $F(x)=-\frac{x^{3}}{30}+250 x$, é uma primitiva de $f$. Então,

$$
\operatorname{Area}(A)=\int_{0}^{40} f(t) d t=F(40)-F(0)=\frac{23600}{3} .
$$

Corolário 2. Sejam as funções $f, g:[a, b] \rightarrow \mathbb{R}$ contínuas e tais que $f(x) \geq g(x) \geq 0, \forall x \in[a, b]$. Então a área da região $A$ compreendida entre os gráficos das funções $f$ e $g$, e as retas $x=a$ e $x=b$ (ver Figura 17), é dada por

$$
\operatorname{Area}(A)=\int_{a}^{b}(f-g)(x) d x \text {. }
$$

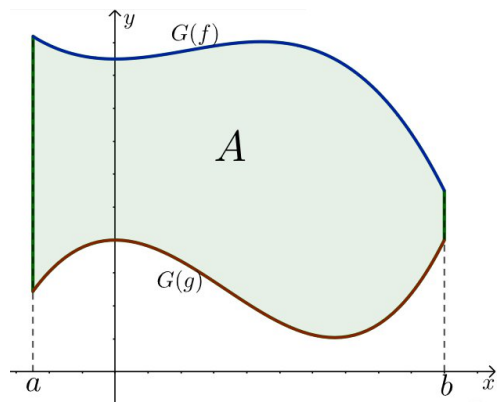

Figura 17: Região $A$ entre os gráficos das funções $f$ e $g$ tais que $f(x), g(x) \geq 0$ Fonte: Autoria própria

\section{Cálculo da Integral Definida usando GeoGebra}

Nesta seção, resolveremos os problemas mencionados na introdução deste artigo, isto é, os problemas de obter estimativas para a área devastada devido ao desastre em Brumadinho (MG) (ver Figura 1), e para a área da Antártida (ver Figura 2), por intermédio do aplicativo de geometria dinâmica, o GeoGebra Clássico 6, que está disponível em [4]. Os principais itens do layout inicial desse aplicativo, ver Figura 18, são: a janela de visualização, a janela de álgebra, o campo de entrada, onde daremos a maioria dos comandos, algumas ferramentas, um teclado virtual, com algumas teclas adicionais, e opções de configuração. 


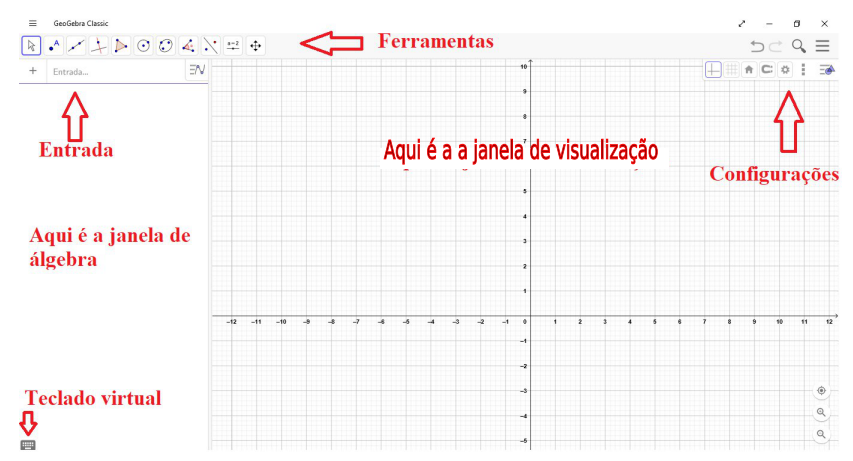

Figura 18: Layout inicial do GeoGebra

Fonte: Autoria própria

\subsection{Plotagem de gráficos de funções}

Usaremos o campo de Entrada para plotagem dos gráficos das funções no aplicativo. Sempre, após a inserção do comando nesse campo, apertaremos a tecla Enter. Por exemplo, se escrever o comando $x \wedge 2$ e, logo após, pressionar essa tecla, veremos que aparecerá $f(x)=x^{2}$ na janela de álgebra e, simultaneamente, o gráfico da função $f: \mathbb{R} \rightarrow \mathbb{R}$, definida por $f(x)=x^{2}$, na janela de visualização (ver Figura 19).

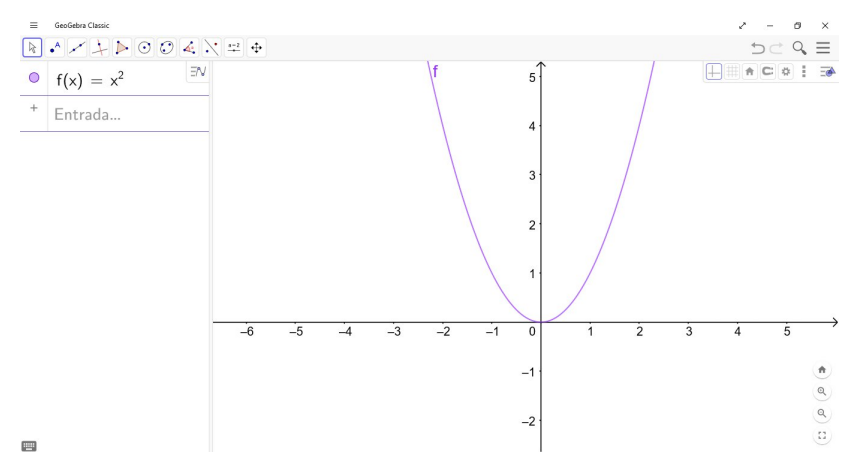

Figura 19: Plotagem do gráfico de $f: \mathbb{R} \rightarrow \mathbb{R}$ definida por $f(x)=x^{2}$

Fonte: Autoria própria

Observe que o próprio software escolheu o domínio da função. Ou seja, se inserirmos a lei que define a imagem de qualquer função, o aplicativo fará a plotagem do gráfico considerando o domínio maximal da função, isto é, o maior subconjunto dos reais no qual a função está definida. Se houver a necessidade de plotar o gráfico de uma função $f$ definida em um intervalo fechado $[a, b]$, podemos utilizar o comando $F u n c ̧ a ̃ o(f, a, b)$, onde $f$ é a expressão que define a função ou é uma função que foi inserida previamente.

Vale ressaltar ainda que o GeoGebra nomeou a função automaticamente. Poderemos, porém, nomear a função como acharmos mais conveniente. Para isso, basta inserir, por exemplo, $f(x)=$, 
seguido da lei que define a função.

Exemplo 6. Façamos a plotagem do gráfico da função $f:[0,2] \rightarrow \mathbb{R}$ definida por $f(x)=x^{2}$. Para isso, basta inserirmos o comando $f(x)=F u n c ̧ \tilde{o} o(x \wedge 2,0,2)$ que aparecerá o gráfico desejado, ver Figura 20.

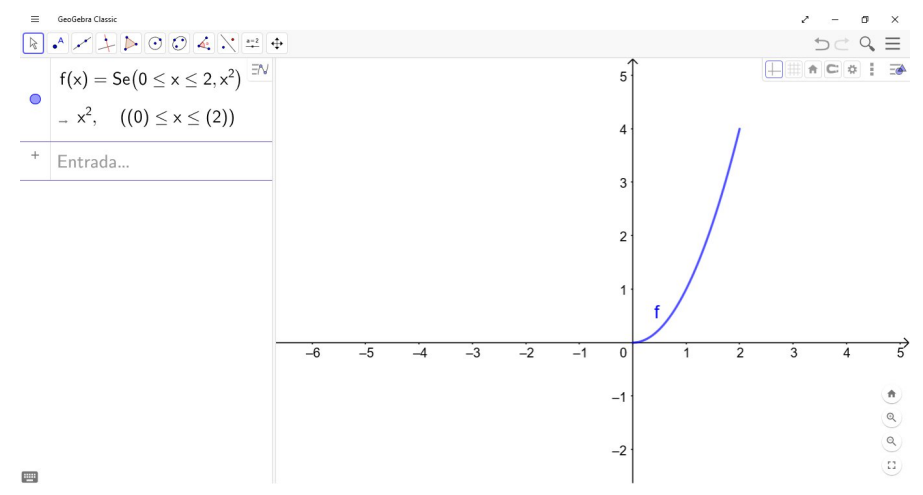

Figura 20: Plotagem do gráfico de $f:[0,2] \rightarrow \mathbb{R}$ definida por $f(x)=x^{2}$ Fonte: Autoria própria

Observação 6. A plotagem do gráfico da função $f$, do Exemplo 6, poderia ser realizada fazendo a inserção do comando $g(x)=x \wedge 2$ e, em seguida, $f(x)=F u n c ̧ a ̃ o(g, 0,2)$.

Como vimos anteriormente, inserindo Função $(f, a, b)$, o programa entenderá como a função $f$ definida no intervalo fechado $[a, b]$. Para intervalos de modo geral, podemos utilizar o comando $S e(<$ condição $>$, $<$ então $>$ ), onde $<$ condição $>$ escreveremos o intervalo para os valores de $x$ e $<$ então $>$ a função $f$ ou a expressão que define a função $f$.

Outra maneira de plotar o gráfico de uma função no GeoGebra é utilizando a ferramenta função à mão livre, ver Figura 21. Como o próprio da ferramenta sugere, desenha-se o gráfico da função diretamente na janela de visualização com o mouse ou, se for o caso, com o toque na tela touchscreen.

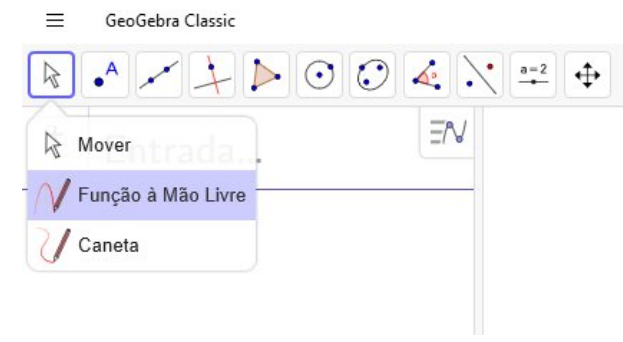

Figura 21: Ferramenta função à mão livre Fonte: Autoria própria

Vale ressaltar que, nesse caso, aparecerá, na janela de álgebra, $f(x)=$ mãolivre $(x)$, ver Figura 22. 
Ou seja, não obteremos a fórmula da função. Mas, ainda sim, será possível calcular a Integral dessas funções, conforme veremos mais adiante.

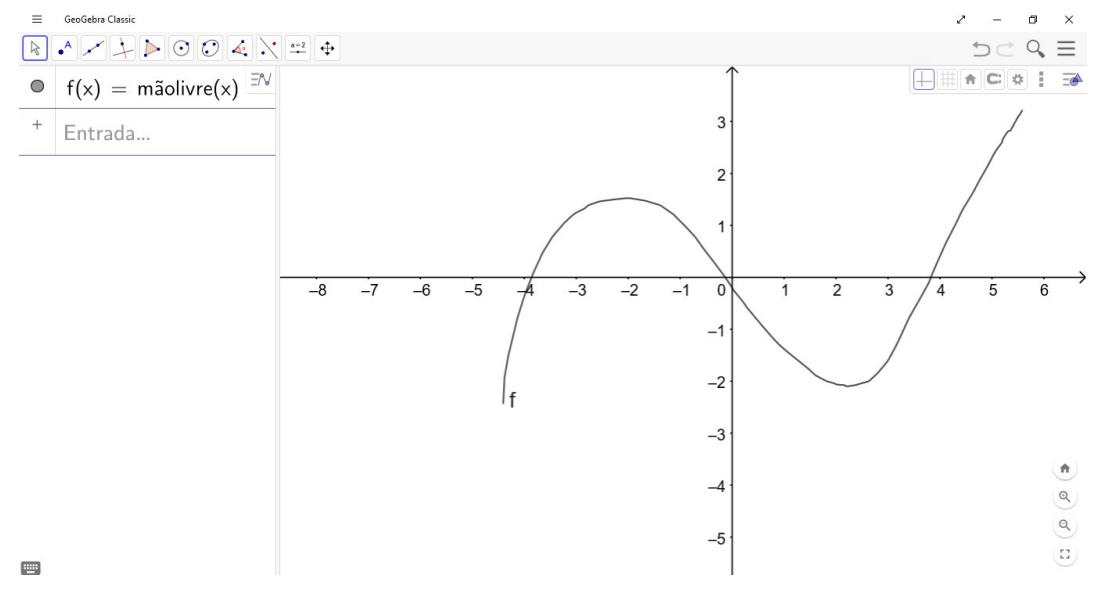

Figura 22: Plotagem do gráfico de uma função mediante a ferramenta função à mão livre

Fonte: Autoria própria

\subsection{Integrais Definidas}

Nesta subseção, apresentaremos outra aplicação interessante do GeoGebra, que é a de calcular e representar geometricamente as integrais definidas.

Para determinarmos o valor e obter uma representação geométrica da Integral definida de uma função $f$ no intervalo $[a, b]$, inseriremos o seguinte comando: $\operatorname{Integral}(f, a, b)$.

Exemplo 7. Seja $f: \mathbb{R} \rightarrow \mathbb{R}$ uma função, definida por $f(x)=-\frac{x^{2}}{10}+250$.

Para determinarmos o valor da Integral $\int_{0}^{50} f(x) d x$ e plotar sua representação geométrica, ver Figura 23, sigamos os passos a seguir:

Passo 1. Faça a inserção do comando $f(x)=-(x \wedge 2) / 10+250$.

Passo 2. Faça a inserção do comando $\operatorname{Integral}(f, 0,50)$. 


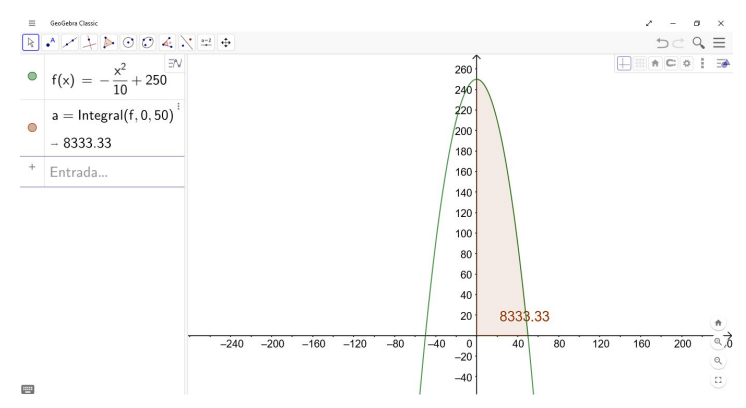

Figura 23: Cálculo e representação geométrica da Integral definida Fonte: Autoria própria

Outro recurso que podemos explorar nesse software é o de calcular e representar a área entre os gráficos das funções $f$ e $g$ no intervalo $[a, b]$, mediante o recurso IntegralEntre $(f, g, a, b)$. Observe que esse recurso representa a integral $\int_{a}^{b}(f-g)(x) d x$.

Exemplo 8. Qual a área da região compreendida entre os gráficos das funções $f, g: \mathbb{R} \rightarrow \mathbb{R}$, definidas por $f(x)=x^{2}$ e $g(x)=2 x+3$, no intervalo $[-1,3]$ ?

Para determinar a área pedida e plotar sua representação geométrica, ver Figura 24, basta inserirmos os comandos a seguir:

Passo 1. Faça a inserção do comando $f(x)=x \wedge 2$.

Passo 2. Faça a inserção do comando $g(x)=2 x+3$.

Passo 3. Faça a inserção do comando IntegralEntre $(g, f,-1,3)$.

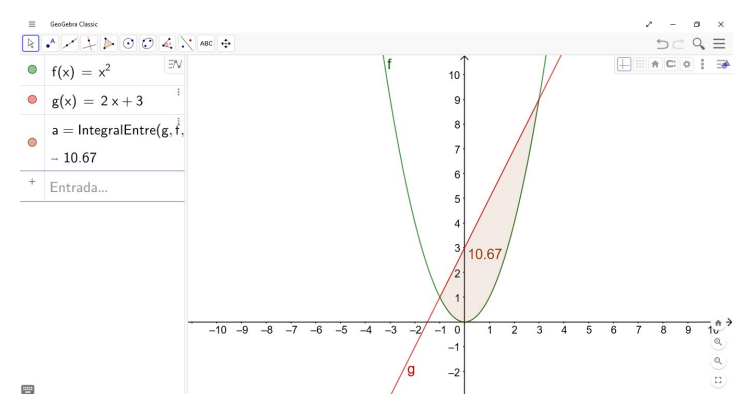

Figura 24: Cálculo e representação geométrica da integral da diferença entre duas funções Fonte: Autoria própria

Observação 7. No Exemplo 8, se inserirmos IntegralEntre(f,g, -1, 3), aparecerá em destaque a mesma região na janela de visualização, mas o valor da integral será negativo, visto que, no intervalo $[-1,3], f(x) \leq g(x)$. Vale ressaltar ainda que a área dessa região é, por definição, o módulo dessa integral. 


\subsection{Recursos complementares}

Nesta subseção, aprenderemos a inserir figuras, realizar configurações básicas da janela de visualização e utilizar as ferramentas Ponto e Segmento.

Para inserir uma figura basta escolher a ferramenta Inserir Imagem, ver Figura 25.

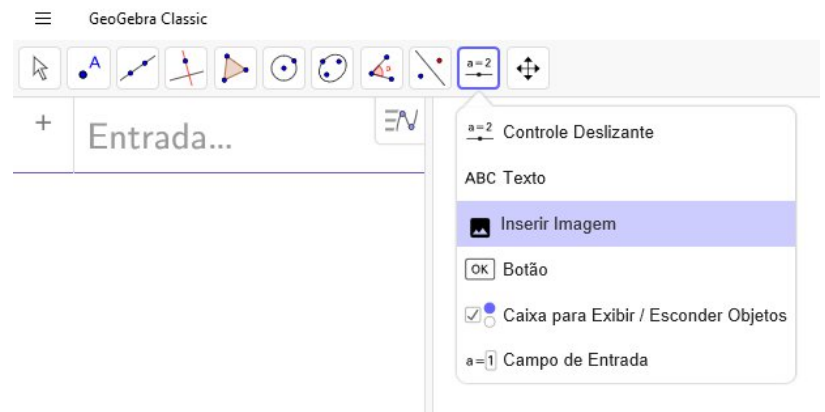

Figura 25: Ferramenta Inserir Imagem

Fonte: Autoria própria

Após escolher essa ferramenta, o aplicativo dará a opção de escolher a imagem armazenada em algum dispositivo ou inserir a imagem mediante foto da webcam.

Quando fizer a inserção da imagem, o programa, por padrão, colocará a imagem como objeto auxiliar, isto é, não aparecerá nenhuma referência na janela de álgebra. Mas, também por padrão, o software mostrará dois pontos vinculados à imagem, onde poderemos transladar, rotacionar, ampliar ou reduzir essa imagem por intermédio do deslocamento desses pontos.

Poderemos definir uma imagem como plano de fundo da janela de visualização. Dessa forma, veremos os eixos e a malha à frente da imagem, ver Figura 26. Para isso, sigamos os passos a seguir:

Passo 1. Dê um clique com o botão esquerdo do mouse sobre a imagem.

Passo 2. Escolha a opção Configurações no canto superior direito da janela de visualização.

Passo 3. Escolha a opção Imagem de Fundo.

Passo 4. Feche a janela de configurações. 


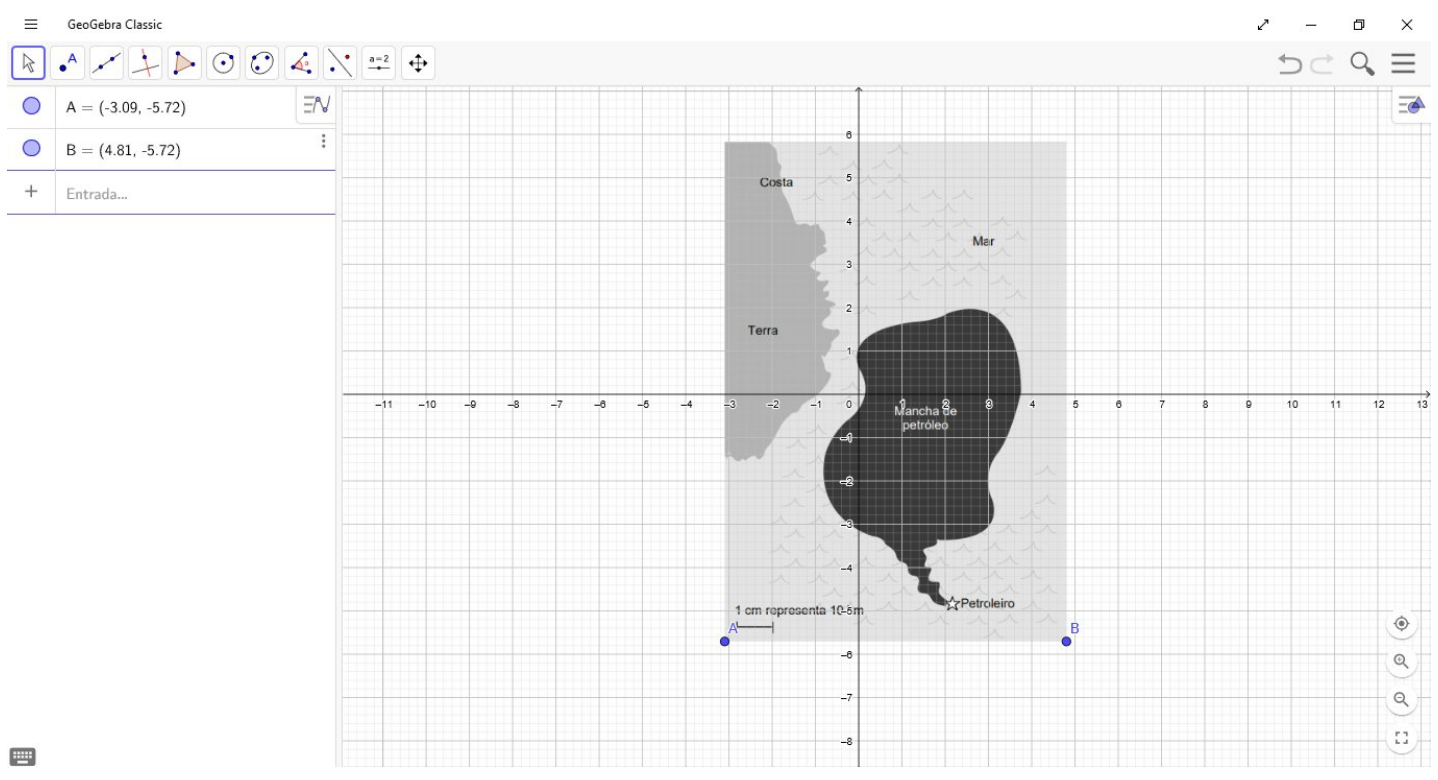

Figura 26: Imagem ao fundo

Fonte: Autoria própria

Outra configuração importante é a dos eixos cartesianos. Para isso, clique com o botão esquerdo do mouse em algum lugar da janela de visualização, onde não há objeto, e escolha a opção Configurações no canto superior direito da janela de visualização. Assim como na configuração da imagem, abrirá uma janela, ver Figura 27. Nessa janela aparecerão varias opções, inclusive a opção de configurar a malha quadriculada.

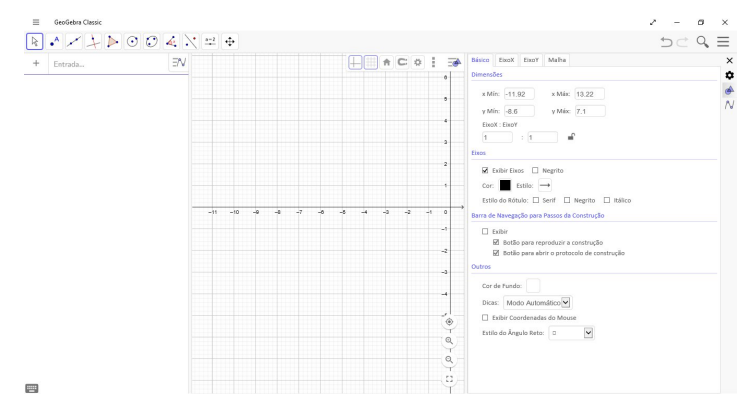

Figura 27: Configurações da janela de visualização

Fonte: Autoria própria

Observação 8. O ícone do cadeado, ao lado da escala, dá a opção de fixar uma determinada escala enquanto trabalhamos com software.

Agora, finalizaremos esta subseção, mostrando o uso das ferramentas Ponto e Segmento que são bastante intuitivas. Ao escolher a opção Ponto, ver Figura 28, dê um clique com o botão esquerdo 
do mouse onde deseja determinar um ponto na janela de visualização. Vale ressaltar ainda que o aplicativo mostrará, também, uma representação do ponto na janela de álgebra.

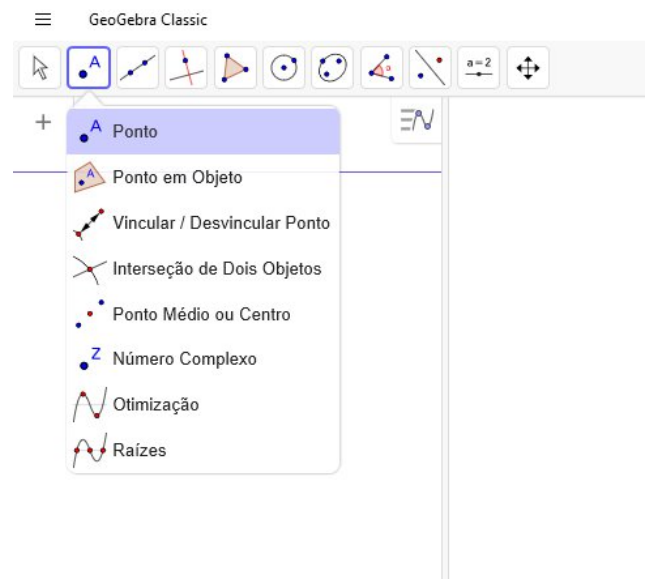

Figura 28: Ferramenta Ponto

Fonte: Autoria própria

Poderemos utilizar a ferramenta Segmento de duas formas: mediante dois pontos dados ou determinando dois pontos. Ao escolher esse recurso, ver Figura 29, determine os extremos do segmento por intermédio de um clique, com o botão esquerdo do mouse, sobre dois pontos existentes ou defina os pontos extremos do segmento. Dessa forma, de imediato, o aplicativo representará, na janela de álgebra, o segmento, evidenciando o comprimento, e os pontos de suas extremidades.

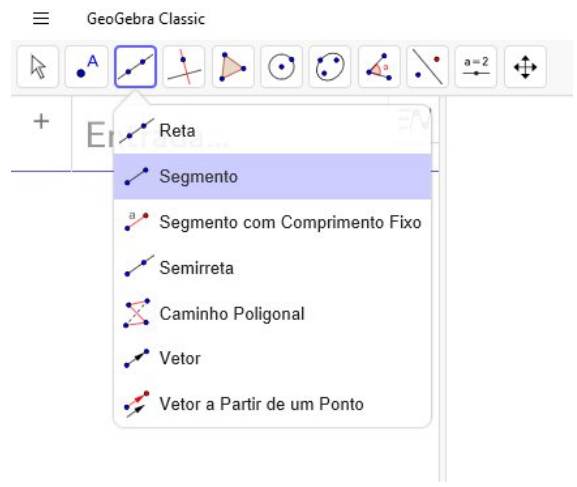

Figura 29: Ferramenta Segmento

Fonte: Autoria própria 


\subsection{Resolução de problemas}

Nesta subseção, mostraremos como utilizar os recursos do GeoGebra para obtermos as estimativas para as áreas da região da Antártida, ver Figura 2, e da região devastada em Brumadinho (MG), ver Figura 1, devido ao rompimento da barragem de uma mineradora.

Inicialmente, daremos uma estimativa para a área da região da Antártida. Para isso, sigamos os passos:

Passo 1. Configure os eixos cartesianos e malha quadriculada com escala de 1 por 1 e fixe essa escala.

Passo 2. Faça inserção da imagem do mapa da Antártida.

Passo 3. Defina o mapa como imagem de fundo.

Passo 4. Rotacione, translade, amplie ou reduza a imagem de tal forma que facilite a plotagem dos gráficos de algumas funções sobre a fronteira da Antártida.

Passo 5. Rotacione, translade, amplie ou reduza a imagem de tal forma que sua escala coincida com a escala dos eixos cartesianos.

Passo 6. Usando a ferramenta função à mão livre, desenhe o gráfico das funções sobre a fronteira da Antártida.

Passo 7. Use o comando IntegralEntre $(f, g, a, b)$, onde $f$ e $g$ são as funções sobre a fronteira da Antártida no intervalo $[a, b]$.

Então, seguindo esses passos e considerando a escala do mapa da Antártida, ver Figura 30, a área pedida será aproximadamente igual a $14.730 .000 \mathrm{~km}^{2}$.

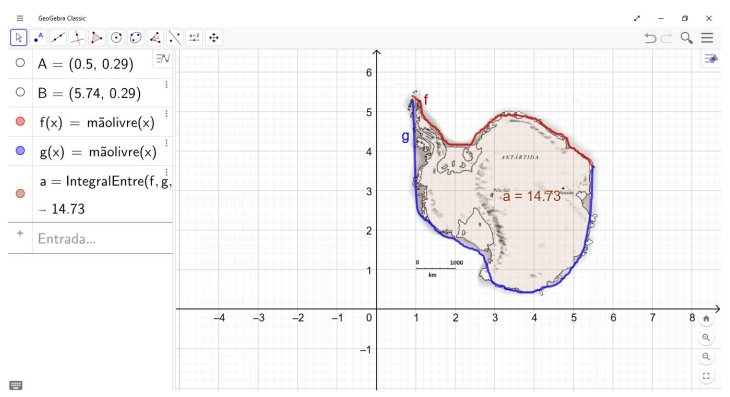

Figura 30: Área da Antártida no GeoGebra Fonte: Autoria própria

Observação 9. Se houver dificuldade em verificar se a escala do mapa está coincidindo com a escala dos eixos, crie um segmento sobre a escala e verifique o comprimento. 
Por último, para obter uma estimativa para a área da região devastada em Brumadinho, seguiremos os passos análogos ao da estimativa da área da Antártida. Ou seja, inseriremos o mapa da região devastada e definiremos como imagem de fundo, posicionaremos a imagem de tal forma que facilite o desenho o gráfico das funções sobre a fronteira da região e que a escala dos eixos coincida com a escala do mapa, usaremos a ferramenta função à mão livre para determinar os gráficos das funções $f$ e $g$ sobre a fronteira no intervalo $[a, b]$ e, finalmente, usaremos o comando IntegralEntre $(f, g, a$, b). Dessa forma, ver Figura 31, a área procurada terá aproximadamente 309 hectares.

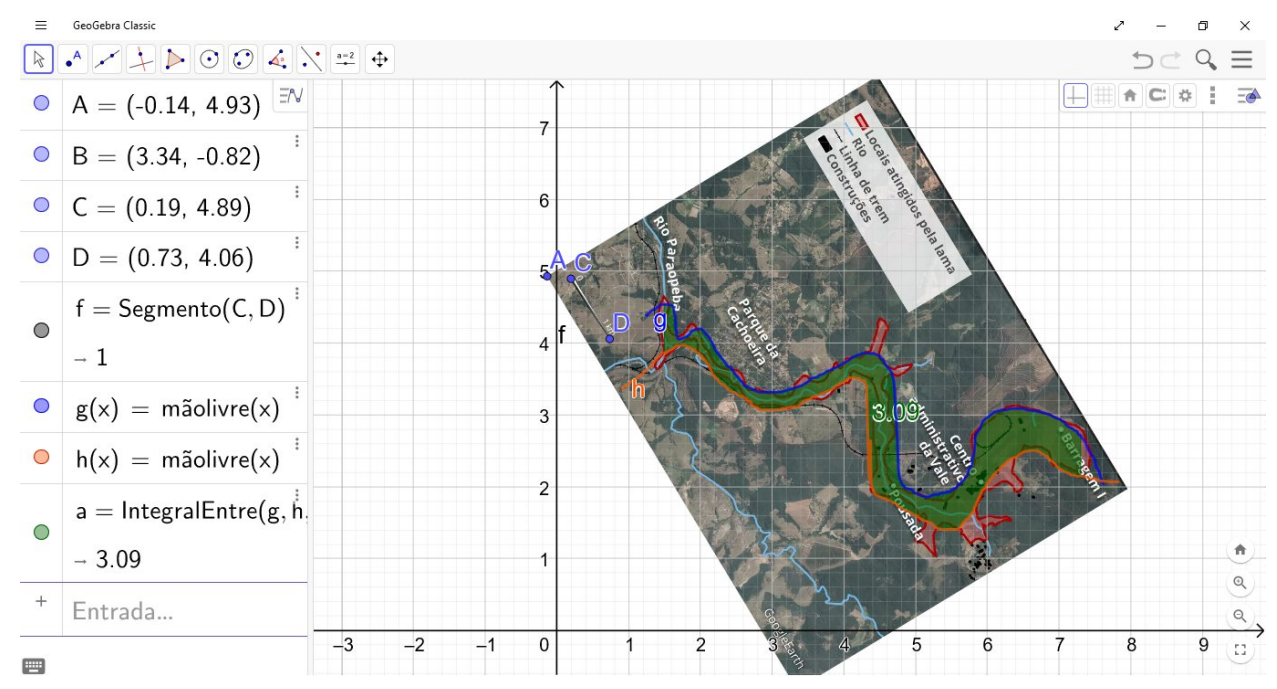

Figura 31: Área da região devastada após rompimento da barragem em Brumadinho (MG) Fonte: Autoria própria

\section{Conclusão}

Por intermédio dos conhecimentos elementares do Cálculo Integral, os alunos do Ensino Médio poderão desenvolver competências e habilidades específicas de Matemática e suas tecnologias esperadas para a etapa final da Educação Básica, segundo a Base Nacional Comum Curricular (BNCC). Além disso, por intermédio do GeoGebra, poderemos propor solução para o problema envolvendo estimativa da área de uma região do plano que não é delimitada apenas por segmentos de retas, como algumas questões dos exames do Programme for International Student Assessment (Pisa).

Vale ressaltar ainda que, nesse texto, apresentamos o Cálculo Integral apelando para intuição geométrica, pois, dessa forma, acreditamos alcançar um número maior de estudantes do Ensino Médio. Para uma abordagem mais completa, inclusive para os conceitos de limite, continuidade e derivada, recomendamos a leitura de [6].

\section{Agradecimentos}

Este artigo é parte do meu Trabalho de Conclusão de Curso (TCC) do Profmat-Ufal. Agradeço ao Prof. Hilário Alencar pela sua orientação. Também sou grato à Larissa Cândido e ao parecerista desse artigo pelos comentários e sugestões. 


\section{Referências}

[1] Brasil. Base Nacional Comum Curricular do Ensino Médio. Brasília, 2018. Disponível em: <http://basenacionalcomum.mec.gov.br/images/BNCC_EI_EF_110518_versaofinal_ site.pdf>. Acesso em: 7 jun. 2019.

[2] Carvalho, P. C. P.; Morgado, A. C. Matemática discreta. $2^{a}$ ed. Rio de Janeiro: SBM, 2015.

[3] Eves, Howard. Introdução à história da matemática. Campinas, SP: Unicamp, 2004.

[4] GeoGebra. Aplicativos matemáticos. Disponível em: <https://www.geogebra.org/>. Acesso em: 7 jun. 2019.

[5] G1, Globo. Tragédia em Brumadinho: o caminho da lama. Disponível em: <https://g1.globo. com/mg/minas-gerais/noticia/2019/01/27/tragedia-em-brumadinho-o-caminho-da-lama.

ghtml>. Acesso em: 7 jun. 2019.

[6] Iezzi, G.; Machado, N. J.; Murakami, C. Fundamentos de Matemática Elementar: limites, derivadas e noções de integral. $7^{a}$ ed. São Paulo: Atual, 2013. v. 8.

[7] Inep. Exemplos de questões do Pisa. Disponível em: <http://portal.inep.gov.br/web/guest/ acoes-internacionais/pisa/provas $>$. Acesso em: 7 abr. 2019.

[8] Lima, E. L. Análise real: funções de uma variável. Rio de Janeiro: Impa, 2017. v. 1.

Alyrio A. Cordeiro Neto EREM Prof. Jerônimo Gueiros 55295-270, Garanhuns, Pernambuco, Brasil $<$ alyrioneto@live.com>

Recebido: $26 / 04 / 2019$

Publicado: 28/06/2019 